\title{
THE $L^{2}$-BOUNDEDNESS OF PSEUDODIFFERENTIAL OPERATORS
}

\author{
I. L. HWANG
}

\begin{abstract}
We give a new proof of the Calderon-Vaillancourt theorem. We also obtain the $L^{2}$-continuity of $a(x, D)$ if its symbol $a(x, \xi)$ satisfies some suitable conditions.
\end{abstract}

Introduction. We consider a pseudodifferential operator $a(x, D)$ on an open set $\Omega \subseteq R^{n}$. We want to find conditions on its symbol $a(x, \xi)$ that imply the $L^{2}$ continuity of $a(x, D)$. Several results for this problem can be found in the literature. We mention a few. The first is due to A. Calderon and R. Vaillancourt [1].

THEOREM. Let $0 \leqslant \rho<1$ and $a: R^{n} \times R^{n} \rightarrow \mathrm{C}$ be a continuous function whose derivatives $\partial_{x}^{\alpha} \partial \xi \xi$ in the distribution sense satisfy the following condition:

There is a constant $C>0$ such that

$$
\left|\partial_{x}^{\alpha} \partial_{\xi}^{\beta} a(x, \xi)\right| \leqslant C(1+|\xi|)^{\rho(|\alpha|-|\beta|)},
$$

where $(x, \xi) \in R^{n} \times R^{n}, 0 \leqslant|\beta| \leqslant 2[n / 2]+n$ and $0 \leqslant|\alpha| \leqslant$ $2 m$ with $m \in N$ and $m(1-\rho) \geqslant \frac{5}{4} n$.

Then $a(x, D)$ is continuous from $L^{2}\left(R^{n}\right)$ to $L^{2}\left(R^{n}\right)$.

Coifman and Meyer [2] improved (*) by assuming that it holds for $0 \leqslant|\alpha|$, $|\beta| \leqslant m, m \in N$ and $m \geqslant[n / 2]+1$. Moreover, they gave an example that shows $m \geqslant[n / 2]+1$ is the best possible. Kato [3], Kumano-go [4], R. Beals [5], Cordes [6] and others obtained similar results by assuming $(*)$ holds for different numbers of derivatives.

In this work we prove several new results about the $L^{2}$ continuity of $a(x, D)$ when $a(x, \xi)$ and some of its derivatives satisfy conditions similar to the above theorem.

$\S 1$ contains our results, and $\S 2-\S 6$ contain the proofs.

We use elementary tools such as integration by parts, the Fourier transform and Parseval's formula.

The author wishes to thank Professors M. S. Baouendi, J. Gerszonowicz and N. Lerner for helpful discussions.

Received by the editors December 12, 1985.

1980 Mathematics Subject Classification (1985 Revision). Primary 35S10. 


\section{Statement of results.}

Theorem 1. Let $\Omega=\prod_{j=1}^{n}\left(s_{j}, t_{j}\right)$ be a bounded open box in $R^{n}$ and $a: \Omega \times R^{n} \rightarrow \mathbf{C}$ a measurable function whose derivatives $\partial_{x}^{\alpha} a$ in the distribution sense satisfy the following condition:

There is a constant $C>0$ such that $\left\|\partial_{x}^{\alpha} a\right\|_{L^{\infty}\left(\Omega \times R^{n}\right)} \leqslant C$, where $\alpha=\left(\alpha_{1}, \ldots, \alpha_{n}\right) \in N^{n}$ with $\alpha_{j}=0$ or 1.

Then $a(x, D)$ is continuous from $L^{2}\left(R^{n}\right)$ to $L^{2}(\Omega)$ with its norm bounded by $C_{n}\left(1+\max _{1 \leqslant j \leqslant n}\left(t_{j}-s_{j}\right)\right)^{n / 2}\left\|\left|\|\mid\|\right.\right.$, where $C_{n}$ is a constant depending only on $n$, and \|\|$a \|$ is the smallest $C$ such that (1.1) holds.

An immediate consequence of Theorem 1 is the following well-known result.

Corollary 1.1. Let $\Omega$ be a bounded open subset of $R^{n}$ and $a: \Omega \times R^{n} \rightarrow \mathrm{C} a$ measurable function whose derivatives $\partial_{x}^{\alpha} a$ in the distribution sense satisfy the following condition:

There is a constant $C>0$ such that $\left\|\partial_{x}^{\alpha} a\right\|_{L^{\infty}\left(\Omega \times R^{n}\right)} \leqslant C$, where $\alpha=\left(\alpha_{1}, \ldots, \alpha_{n}\right) \in N^{n}$ with $\alpha_{j}=0$ or 1 .

Then $a(x, D)$ is continuous from $L^{2}\left(R^{n}\right)$ to $L_{\mathrm{loc}}^{2}(\Omega)$.

THEOREM 2. Let a: $R^{n} \times R^{n} \rightarrow \mathrm{C}$ be a continuous function whose derivatives $\partial_{x}^{\alpha} \partial \xi \xi$ in the distribution sense satisfy the following condition:

There is a constant $C>0$ such that $\left\|\partial_{x}^{\alpha} \partial_{\xi}^{\beta} a\right\|_{L^{\infty}\left(R^{n} \times R^{n}\right)} \leqslant C$, where $\alpha=\left(\alpha_{1}, \ldots, \alpha_{n}\right), \beta=\left(\beta_{1}, \ldots, \beta_{n}\right) \in N^{n}$ with $\alpha_{j}=0$ or $1, \beta_{j}=0$ or 1 .

Then $a(x, D)$ is continuous from $L^{2}\left(R^{n}\right)$ to $L^{2}\left(R^{n}\right)$ with its norm bounded by $C_{n}\|a\| \|$, where $C_{n}$ is a constant depending only on $n$ and $\||a \||$ is the smallest $C$ such that (1.3) holds.

Remark. We mention that Theorem 2 is also obtained by Coifman and Meyer [2] and by Cordes [6]. We include it because we need it for the proofs of the next results, and because we believe our proof is simpler.

Corollary 2.1. Let a: $R^{n} \times R^{n} \rightarrow \mathrm{C}$ be a measurable function whose derivatives $\partial \xi \beta a$ in the distribution sense satisfy the following condition:

There is a constant $C>0$ such that

$$
\sup _{x \in R^{n}}\left(\int_{R^{n}}\left|\partial_{\xi}^{\beta} a(x, \xi)\right|^{2} d \xi\right)^{1 / 2} \leqslant C,
$$

where $\beta=\left(\beta_{1}, \ldots, \beta_{n}\right) \in N^{n}$ with $\beta_{j}=0$ or 1 .

Then $a(x, D)$ is continuous from $L^{2}\left(R^{n}\right)$ to $L^{2}\left(R^{n}\right)$ with its norm bounded by $C_{n}\|\mid\| a \|$, where $C_{n}$ is a constant depending only on $n$, and $\|a\| \|$ is the smallest $C$ such that (1.4) holds.

REMARK. A different sufficient condition for Corollary 2.1 can be found in Muramoto and Nagase [7]. 
COROllaRy 2.2. Let $a: R^{n} \times R^{n} \rightarrow \mathrm{C}$ be a measurable function whose derivatives $\partial_{x}^{\alpha} a$ in the distribution sense satisfy the following condition:

There is a constant $C>0$ such that

$$
\sup _{\xi \in R^{n}}\left(\int_{R^{n}}\left|\partial_{x}^{\alpha} a(x, \xi)\right|^{2} d x\right)^{1 / 2} \leqslant C,
$$

where $\alpha=\left(\alpha_{1}, \ldots, \alpha_{n}\right) \in N^{n}$ with $\alpha_{j}=0$ or 1 .

Then $a(x, D)$ is continuous from $L^{2}\left(R^{n}\right)$ to $L^{2}\left(R^{n}\right)$ with its norm bounded by $C_{n}\|\mid a\| \|$, where $C_{n}$ is a constant depending only on $n$, and $\||a|\|$ is the smallest $C$ such that (1.5) holds.

REMARK. A different sufficient condition for Corollary 2.2 can be found in Hörmander [8].

THEOREM 3. Let $\rho \in R, \rho<1$, and $a: R^{n} \times R^{n} \rightarrow \mathrm{C}$ be a continuous function whose derivatives $\partial_{x}^{\alpha} \partial \xi \xi$ in the distribution sense satisfy the following condition:

There is a constant $C>0$ such that

$$
\left|\partial_{x}^{\alpha} \partial_{\xi}^{\beta} a(x, \xi)\right| \leqslant C(1+|\xi|)^{\rho(|\alpha|-|\beta|)},
$$

where $(x, \xi) \in R^{n} \times R^{n}, \alpha=\left(\alpha_{1}, \ldots, \alpha_{n}\right), \beta=\left(\beta_{1}, \ldots, \beta_{n}\right)$

$\in N^{n}$ with $\alpha_{j}=0,1$ or $2, \beta_{j}=0$ or 1 .

Then $a(x, D)$ is continuous from $L^{2}\left(R^{n}\right)$ to $L^{2}\left(R^{n}\right)$ with its norm bounded by $C_{\rho, n}\||a|\|$, where $C_{\rho, n}$ is a constant depending only on $\rho$ and $n$, and $\|\mid a\|$ is the smallest C such that (1.6) holds.

REMARK. When $-\infty<\rho<1$, a different sufficient condition for Theorem 3 can be found in R. Beals [5].

THEOREM 4. Let $g_{t}(\xi)=(\log (1+|\xi|))^{-t}, t>0$. Suppose $a: R^{n} \times R^{n} \rightarrow \mathrm{C}$ is $a$ continuous function whose derivatives $\partial_{x}^{\alpha} \partial \xi a$ in the distribution sense satisfy the following condition:

There is a constant $C>0$ such that

$$
\begin{gathered}
\left|\partial_{x}^{\alpha} \partial_{\xi}^{\beta} a(x, \xi)\right| \leqslant C\left(g_{t}(\xi)(1+|\xi|)\right)^{|\alpha|-|\beta|}, \\
\text { where }(x, \xi) \in R^{n} \times R^{n}, \alpha=\left(\alpha_{1}, \ldots, \alpha_{n}\right), \beta=\left(\beta_{1}, \ldots, \beta_{n}\right) \\
\in N^{n} \text { with } 0 \leqslant \alpha_{j} \leqslant[(4+t) / 2 t]+2 \text { and } \beta_{j}=0 \text { or } 1 .
\end{gathered}
$$

Then $a(x, D)$ is continuous from $L^{2}\left(R^{n}\right)$ to $L^{2}\left(R^{n}\right)$ with its norm bounded by $C_{t, n}\||a|\|$, where $C_{t, n}$ is a constant depending only on $t$ and $n$, and $\||a|\|$ is the smallest $C$ such that (1.7) holds.

TheOREM 5. Let $\rho \in R, \rho<1$. Suppose $a: R \times R \rightarrow \mathbf{C}$ is a continuous function whose derivatives $\partial_{x}^{\alpha} \partial_{\xi}^{\beta}$ a in the distribution sense satisfy the following condition:

There is a constant $C>0$ such that

$$
\left|\partial_{x}^{\alpha} \partial_{\xi}^{\beta} a(x, \xi)\right| \leqslant C(1+|\xi|)^{\rho(\alpha-\beta)},
$$

where $(x, \xi) \in R \times R, \alpha=0$ or $1, \beta=0$ or 1 . 
Then $a(x, D)$ is continuous from $L^{2}(R)$ to $L^{2}(R)$ with its norm bounded by $C_{\rho}\|l\|$, where $C_{\rho}$ is a constant depending only on $\rho$, and $\|a\| \|$ in the smallest $C$ such that (1.8) holds.

Corollary 5.1. Let $g_{t}(\xi)=(\log (1+|\xi|))^{-t}, \xi \in R, t>0$. Suppose $a: R \times R \rightarrow$ $\mathrm{C}$ is a continuous function whose derivatives $\partial_{x}^{\alpha} \partial \xi \beta$ in the distribution sense satisfy the following condition:

There is a constant $C>0$ such that

$$
\left|\partial_{x}^{\alpha} \partial_{\xi}^{\beta} a(x, \xi)\right| \leqslant C\left(g_{t}(\xi)(1+|\xi|)\right)^{\alpha-\beta},
$$

where $(x, \xi) \in R \times R, \alpha, \beta \in N$ and $0 \leqslant \alpha \leqslant[(4+t) / 2 t]$

$+1, \beta=0$ or 1 .

Then $a(x, D)$ is continuous from $L^{2}(R)$ to $L^{2}(R)$ with its norm bounded by $C_{t}\|\mid\| a \|$, where $C_{t}$ is a constant depending only on $t$, and $\||a|\|$ in the smallest $C$ such that (1.9) holds.

2. Proof of Theorem 1. To prove Theorem 1, we need the following lemmas.

Lemma 2.1. If $f(\xi)=1 /(1+i \xi), \xi \in R$, then the Fourier transform of $f$ is

$$
\hat{f}(t)= \begin{cases}0, & t>0, \\ 2 \pi e^{-|t|}, & t<0 .\end{cases}
$$

Proof. Making use of the residue theorem, the reader can check it without difficulty.

More generally, we have

LEMMA 2.2. If

$$
f(\xi)=\prod_{j=1}^{n} \frac{1}{1+i \xi_{j}}, \quad \xi=\left(\xi_{1}, \ldots, \xi_{n}\right) \in R^{n},
$$

then the Fourier transform of $f$ is

$$
\hat{f}(t)=\prod_{j=1}^{n} g_{j}\left(t_{j}\right)
$$

where $t=\left(t_{1}, \ldots, t_{n}\right) \in R^{n}$ and

$$
g_{j}\left(t_{j}\right)= \begin{cases}0, & t_{j}>0, \\ 2 \pi e^{-\left|t_{j}\right|}, & t_{j}<0 .\end{cases}
$$

In the following proposition we shall prove Theorem 1 in the special case $n=1$ and under stronger conditions.

Proposition 2.1. Let $\Omega=(s, t),-\infty<s<t<\infty$. Suppose $a: \Omega \times R \rightarrow \mathbf{C}$ is a continuous function whose derivatives $\partial_{x}^{\alpha}$ a satisfy the following conditions:

(2.1) $\partial_{x}^{\alpha} a \in C^{0}(\Omega \times R), \alpha=0,1$.

(2.2) There is a constant $C>0$ such that $\left\|\partial_{x}^{\alpha} a\right\|_{L^{\infty}(\Omega \times R)} \leqslant C, \alpha=0,1$.

Then $a(x, D)$ is continuous from $L^{2}(R)$ to $L^{2}(\Omega)$ with its norm bounded by $C_{\Omega}\|\| a \|$, where $C_{\Omega}$ is a constant depending only on $\Omega$ and $\|\mid\|$ al is the smallest $C$ such that (2.2) holds. 
Proof. Let $0<\varepsilon<\frac{1}{4}(s-t)$ and $\Omega_{\varepsilon}=(s+\varepsilon, t-\varepsilon)$. Our goal is to find the existence of an absolute constant $C_{0}>0$ such that

$$
\|a(x, D) u\|_{L^{2}\left(\Omega_{\varepsilon}\right)} \leqslant C_{0}(1+t-s-2 \varepsilon)^{1 / 2}\|a\|\|\| u \|_{L^{2}(R)} \quad \forall u \in C_{0}^{\infty}(R) .
$$

Then the proof of Proposition 2.1 follows by letting $\varepsilon \rightarrow 0$.

For $u \in C_{0}^{\infty}(R)$ we have

$$
a(x, D) u(x)=\frac{1}{2 \pi} \int_{R} e^{i x \xi} a(x, \xi) \hat{u}(\xi) d \xi, \quad x \in \Omega .
$$

Therefore, we have

$$
\begin{aligned}
\int_{\Omega_{\varepsilon}} \mid a(x, D) & \left.u(x)\right|^{2} d x \\
= & \left(\frac{1}{2 \pi}\right)^{2} \int_{\Omega_{\varepsilon}} \int_{R} \int_{R} e^{i x(\xi-\lambda)} a(x, \xi) \hat{u}(\xi) \bar{a}(x, \lambda) \overline{\hat{u}}(\lambda) d \xi d \lambda d x \\
= & \left(\frac{1}{2 \pi}\right)^{2} \int_{\Omega_{\varepsilon}} \int_{R} \int_{R} e^{i x \xi} a(x, \xi+\lambda) \hat{u}(\xi+\lambda) \bar{a}(x, \lambda) \overline{\hat{u}}(\lambda) d \xi d \lambda d x .
\end{aligned}
$$

Because the integrand is in $L^{1}\left(\Omega_{\varepsilon} \times R \times R\right)$, by Fubini's theorem we have

$$
\begin{aligned}
& \int_{\Omega_{\varepsilon}}|a(x, D) u(x)|^{2} d x \\
& \quad=\left(\frac{1}{2 \pi}\right)^{2} \int_{R} \int_{R}\left(\int_{\Omega_{\varepsilon}} e^{i x \xi} a(x, \xi+\lambda) \bar{a}(x, \lambda) d x\right) \hat{u}(\xi+\lambda) \overline{\hat{u}}(\lambda) d \xi d \lambda .
\end{aligned}
$$

Making use of the identity

$$
\frac{1}{1+i \xi}\left(1+\partial_{x}\right)\left(e^{i x \xi}\right)=e^{i x \xi}
$$

we obtain

$$
\begin{aligned}
\int_{\Omega_{\varepsilon}} e^{i x \xi} a(x, \xi+\lambda) \bar{a}(x, \lambda) d x= & \frac{1}{1+i \xi} \int_{\Omega_{\varepsilon}} e^{i x \xi} a(x, \xi+\lambda) \bar{a}(x, \lambda) d x \\
& +\frac{1}{1+i \xi} e^{i(t-\varepsilon) \xi} a(t-\varepsilon, \xi+\lambda) \bar{a}(t-\varepsilon, \lambda) \\
& -\frac{1}{1+i \xi} e^{i(s+\varepsilon) \xi} a(s+\varepsilon, \xi+\lambda) \bar{a}(s+\varepsilon, \lambda) \\
& -\frac{1}{1+i \xi} \int_{\Omega_{\varepsilon}} e^{i x \xi} \partial_{x} a(x, \xi+\lambda) \bar{a}(x, \lambda) d x \\
& -\frac{1}{1+i \xi} \int_{\Omega_{\varepsilon}} e^{i x \xi} a(x, \xi+\lambda) \partial_{x} \bar{a}(x, \lambda) d x
\end{aligned}
$$


Substituting (2.4) into (2.3), we have

$$
\begin{aligned}
& \int_{\Omega_{\varepsilon}}|a(x, D) u(x)|^{2} d x \\
&=\left(\frac{1}{2 \pi}\right)^{2} \int_{\Omega_{\varepsilon}} \int_{R} \int_{R} \frac{e^{i x \xi}}{1+i \xi} a(x, \xi+\lambda) \hat{u}(\xi+\lambda) \bar{a}(x, \lambda) \overline{\hat{u}}(\lambda) d \lambda d \xi d x \\
&+\left(\frac{1}{2 \pi}\right)^{2} \int_{R} \int_{R} \frac{e^{i(t-\varepsilon) \xi}}{1+i \xi} a(t-\varepsilon, \xi+\lambda) \hat{u}(\xi+\lambda) \bar{a}(t-\varepsilon, \lambda) \overline{\hat{u}}(\lambda) d \lambda d \xi \\
&-\left(\frac{1}{2 \pi}\right)^{2} \int_{R} \int_{R} \frac{e^{i(s+\varepsilon) \xi}}{1+i \xi} a(s+\varepsilon, \xi+\lambda) \hat{u}(\xi+\lambda) \bar{a}(s+\varepsilon, \lambda) \overline{\hat{u}}(\lambda) d \lambda d \xi \\
&-\left(\frac{1}{2 \pi}\right)^{2} \int_{\Omega_{\varepsilon}} \int_{R} \int_{R} \frac{e^{i x \xi}}{1+i \xi} \partial_{x} a(x, \xi+\lambda) \hat{u}(\xi+\lambda) \bar{a}(x, \lambda) \overline{\hat{u}}(\lambda) d \lambda d \xi d x \\
&-\left(\frac{1}{2 \pi}\right)^{2} \int_{\Omega_{\varepsilon}} \int_{R} \int_{R} \frac{e^{i x \xi}}{1+i \xi} a(x, \xi+\lambda) \hat{u}(\xi+\lambda) \partial_{x} \bar{a}(x, \lambda) \overline{\hat{u}}(\lambda) d \lambda d \xi d x \\
&= I_{1}+I_{2}+I_{3}+I_{4}+I_{5} .
\end{aligned}
$$

We first estimate $I_{1}$.

(2.6) $I_{1}=\left(\frac{1}{2 \pi}\right)^{2} \int_{\Omega_{\varepsilon}} \int_{R} \int_{R} \frac{e^{i x \xi}}{1+i \xi} a(x, \xi+\lambda) \hat{u}(\xi+\lambda) \bar{a}(x, \lambda) \overline{\hat{u}}(\lambda) d \lambda d \xi d x$.

For $x$ fixed we study the following integral:

$$
\begin{aligned}
J_{1} & =\int_{R} \int_{R} \frac{e^{i x \xi}}{1+i \xi} a(x, \xi+\lambda) \hat{u}(\xi+\lambda) \bar{a}(x, \lambda) \overline{\hat{u}}(\lambda) d \lambda d \xi \\
& =\int_{R} g(\xi) h(\xi) d \xi
\end{aligned}
$$

where $g(\xi)=e^{i x \xi} /(1+i \xi)$ and

$$
h(\xi)=\int_{R} a(x, \xi+\lambda) \hat{u}(\xi+\lambda) \bar{a}(x, \lambda) \overline{\hat{u}}(\lambda) d \lambda .
$$

Looking at the expression of $h(\xi)$ and taking into account that $u \in C_{0}^{\infty}(R)$ and $\|a(x, \cdot)\|_{L^{\infty}(R)} \leqslant\||a|\|$, we see that $h$ is a kind of convolution of two functions both belonging to $L^{1}(R) \cap L^{2}(R)$. Therefore we have

$$
h \text { is in } L^{2}(R) \text {, }
$$

and

(2.8) There is an absolute constant $C_{1}>0$ such that

$$
\|\hat{\bar{h}}\|_{L^{1}(R)} \leqslant C_{1}\|a(x, \cdot)\|_{L^{\infty}(R)}^{2}\|u\|_{L^{2}(R)}^{2} \leqslant C_{1}\|a\|^{2}\|u\|_{L^{2}(R)}^{2}
$$


Obviously, $g$ is in $L^{2}(R)$. Making use of Parseval's formula, (2.7), (2.8) and Lemma 2.1, we have

$$
\begin{aligned}
\left|J_{1}\right| & =\left|\int_{R} g(\xi) h(\xi) d \xi\right|=\frac{1}{2 \pi}\left|\int_{R} \hat{g}(\alpha) \overline{\bar{h}}(\alpha) d \alpha\right| \\
& \leqslant \frac{1}{2 \pi}\|\hat{g}\|_{L^{\infty}(R)}\|\hat{h}\|_{L^{1}(R)} \\
& \leqslant C_{2}\|a\|\left\|^{2}\right\| u \|_{L^{2}(R)}^{2} \quad \text { for some absolute constant } C_{2} .
\end{aligned}
$$

Now, going back to (2.6), we obtain

$$
\left|I_{1}\right| \leqslant C_{2}(t-s-2 \varepsilon)\|a\|^{2}\|u\|_{L^{2}(R)}^{2} .
$$

By similar arguments, we have the following estimates:

$$
\begin{aligned}
& \left|I_{2}\right| \leqslant C_{2} \mid\|a\|\left\|^{2}\right\| u \|_{L^{2}(R)}^{2}, \\
& \left|I_{3}\right| \leqslant C_{2}\|a\|\left\|^{2}\right\| u \|_{L^{2}(R),}^{2}, \\
& \left|I_{4}\right| \leqslant C_{2}(t-s-2 \varepsilon)\|a\|^{2}\|u\|_{L^{2}(R)}^{2}
\end{aligned}
$$

and

$$
\left|I_{5}\right| \leqslant C_{2}(t-s-2 \varepsilon)\|a\|^{2}\|u\|_{L^{2}(R)}^{2} .
$$

Thus, it follows from (2.5), (2.9) and (2.10) that

$$
\|a(x, D) u\|_{L^{2}\left(\Omega_{\varepsilon}\right)} \leqslant C_{3}(1+t-s-2 \varepsilon)^{1 / 2}\|a\|\|u\|_{L^{2}(R)} \quad \forall u \in C_{0}^{\infty}(R),
$$

where $C_{3}$ is an absolute constant.

More generally, we have

Proposition 2.2. Let $\Omega=\prod_{j=1}^{n}\left(s_{j}, t_{j}\right)$ be a bounded open box in $R^{n}$. Suppose a: $\Omega \times R^{n} \rightarrow \mathbf{C}$ is a continuous function whose derivatives $\partial_{x}^{\alpha} a$ satisfy the following conditions:

$$
\partial_{x}^{\alpha} a \in C^{0}\left(\dot{\Omega} \times R^{n}\right), \quad \alpha=\left(\alpha_{1}, \ldots, \alpha_{n}\right), \alpha_{j}=0 \text { or } 1 .
$$

(2.12) There is a constant $C>0$ such that

$$
\left\|\partial_{x}^{\alpha} a\right\|_{L^{\infty}\left(\Omega \times R^{n}\right)} \leqslant C, \quad \alpha=\left(\alpha_{1}, \ldots, \alpha_{n}\right), \alpha_{j}=0 \text { or } 1 .
$$

Then $a(x, D)$ is continuous from $L^{2}\left(R^{n}\right)$ to $L^{2}(\Omega)$ with its norm bounded by $C_{n}\left(1+\max _{1 \leqslant j \leqslant n}\left(t_{j}-s_{j}\right)\right)^{n / 2}\|a\| \|$, where $C_{n}$ is a constant depending only on $n$, and IIa || is the smallest $C$ such that (2.12) holds.

Proof. The proposition follows from Lemma 2.2 and an argument similar to the proof of Proposition 2.1.

Now, we are ready to prove Theorem 1.

ProOF OF THEOREM 1. We shall approach the symbol a by smooth symbols. Then we shall apply Proposition 2.2.

Let $\varphi$ be in $C_{0}^{\infty}(R)$ such that the following conditions hold:

$$
\varphi \geqslant 0, \quad \operatorname{supp} \varphi \subseteq\{-1 \leqslant y \leqslant 1\} \text { and }\|\varphi\|_{L^{1}(R)}=1 .
$$


If $0<\varepsilon<\frac{1}{8} \min _{1 \leqslant j \leqslant n}\left(t_{j}-s_{j}\right)$, then we define $a_{\varepsilon}=\lambda_{\varepsilon} * a$, where

$$
\lambda_{\varepsilon}(x, \xi)=\frac{1}{\varepsilon^{2 n}} \prod_{j=1}^{n} \varphi\left(\frac{x_{j}}{\varepsilon}\right) \varphi\left(\frac{\xi_{j}}{\varepsilon}\right),
$$

with $x=\left(x_{1}, \ldots, x_{n}\right), \xi=\left(\xi_{1}, \ldots, \xi_{n}\right) \in R^{n}$. We see that $a_{\varepsilon} \in C^{\infty}\left(R^{n} \times R^{n}\right)$ and $\lim _{\varepsilon \rightarrow 0} a_{\varepsilon}(x, \xi)=a(x, \xi)$ a.e. in $\Omega \times R^{n}$. Moreover, hypothesis (1.1) gives

$$
\left\|\partial_{x}^{\alpha} a_{\varepsilon}\right\|_{L^{\infty}\left(\Omega_{\varepsilon_{0}} \times R^{n}\right)} \leqslant\|a\| \|
$$

where $0<\varepsilon \leqslant \varepsilon_{0}, \Omega_{\varepsilon_{0}}=\prod_{j=1}^{n}\left(s_{j}+2_{\varepsilon_{0}}, t_{j}-2 \varepsilon_{0}\right)$ and $\alpha=\left(\alpha_{1}, \ldots, \alpha_{n}\right) \in N^{n}$ with $\alpha_{j}=0$ or 1. By applying Proposition 2.2 to $a_{\varepsilon}(x, D) u$ with $u \in C_{0}^{\infty}\left(R^{n}\right)$, we get for $0<\varepsilon \leqslant \varepsilon_{0}$

$$
\left\|a_{\varepsilon}(x, D) u\right\|_{L^{2}\left(\Omega_{\varepsilon_{0}}\right)} \leqslant C_{n}\left(1-4_{\varepsilon_{0}}+\max _{1 \leqslant j \leqslant n}\left(t_{j}-s_{j}\right)\right)^{n / 2}\|a\|\|u\|_{L^{2}\left(R^{n}\right)}
$$

We now write

$$
\begin{aligned}
\| a_{\varepsilon}(x, D) & u \|_{L^{2}\left(\Omega_{\tau_{0}}\right)}^{2} \\
& =\left(\frac{1}{2 \pi}\right)^{2 n} \int_{\Omega_{\varepsilon_{0}}} \int_{R^{n}} \int_{R^{n}} e^{i x(\xi-\lambda)} a_{\varepsilon}(x, \xi) \hat{u}(\xi) \bar{a}_{\varepsilon}(x, \lambda) \overline{\hat{u}}(\lambda) d \lambda d \xi d x .
\end{aligned}
$$

Then by the facts $\lim _{\varepsilon \rightarrow 0} a_{\varepsilon}=a$ a.e. in $\Omega \times R^{n},(2.14), \hat{u} \in S\left(R^{n}\right),\left|\Omega_{\varepsilon_{0}}\right|<\infty$, and the Lebesgue dominated convergence theorem, we obtain

$$
\begin{aligned}
\lim _{\varepsilon \rightarrow 0}\left\|a_{\varepsilon}(x, D) u\right\|_{L^{2}\left(\Omega_{\varepsilon_{0}}\right)}^{2} & \\
= & \left(\frac{1}{2 \pi}\right)^{2 n} \lim _{\varepsilon \rightarrow 0} \int_{\Omega_{\varepsilon_{0}}} \int_{R^{n}} \int_{R^{n}} e^{i x(\xi-\lambda)} a_{\varepsilon}(x, \xi) \hat{u}(\xi) \bar{a}_{\varepsilon}(x, \lambda) \overline{\hat{u}}(\lambda) d \lambda d \xi d x \\
& =\left(\frac{1}{2 \pi}\right)^{2 n} \int_{\Omega_{\varepsilon_{0}}} \int_{R^{n}} \int_{R^{n}} e^{i x(\xi-\lambda)} a(x, \xi) \hat{u}(\xi) \bar{a}(x, \lambda) \overline{\hat{u}}(\lambda) d \lambda d \xi d x \\
& =\|a(x, D) u\|_{L^{2}\left(\Omega_{\tau_{0}}\right) .}^{2} .
\end{aligned}
$$

This and (2.15) imply

$$
\|a(x, D) u\|_{L^{2}\left(\Omega_{t_{0}}\right)} \leqslant C_{n}\left(1-4 \varepsilon_{0}+\max _{1 \leqslant j \leqslant n}\left(t_{j}-s_{j}\right)\right)^{n / 2}\|a\|\|\| u \|_{L^{2}\left(R^{n}\right) .}
$$

Then Theorem 1 follows by letting $\varepsilon_{0} \rightarrow 0$.

Proof of Corollary 1.1. Since any compact subset of $\Omega$ can be covered by a union of finite open boxes in $\Omega$, Corollary 1.1 follows from Theorem 1.

3. Proof of Theorem 2. To prove Theorem 2, we need the following crucial lemma which is related to Wigner functions.

Lemma 3.1. For $u, \varphi \in L^{2}\left(R^{n}\right)$ we define

$$
g(x, \xi)=\int_{R^{n}} e^{-i y \xi} \varphi(x-y) u(y) d y,
$$


and

$$
h(x, \xi)=\int_{R^{n}} e^{i x y} \varphi(\xi+y) u(y) d y, \quad(x, \xi) \in R^{n} \times R^{n} .
$$

Then we have

$$
\|g\|_{L^{2}\left(R^{n} \times R^{n}\right)}=\|h\|_{L^{2}\left(R^{n} \times R^{n}\right)}=(2 \pi)^{n / 2}\|\varphi\|_{L^{2}\left(R^{n}\right)}\|u\|_{L^{2}\left(R^{n}\right) .}
$$

PRoOF. Without loss of generality, we can assume that $u, \varphi \in C_{0}^{\infty}\left(R^{n}\right)$. Making use of Parseval's formula, we have

$$
\int_{R^{n}} \int_{R^{n}}|g(x, \xi)|^{2} d x d \xi=\left(\frac{1}{2 \pi}\right)^{n} \int_{R^{n}} \int_{R^{n}}|\widehat{g(\cdot, \xi)}(\alpha)|^{2} d \alpha d \xi
$$

By using the identity $\overline{g(\cdot, \xi)}(\alpha)=\hat{\varphi}(\alpha) \hat{u}(\alpha+\xi)$ and Fubini's theorem, we obtain

$$
\|g\|_{L^{2}\left(R^{n} \times R^{n}\right)}=(2 \pi)^{n / 2}\|\varphi\|_{L^{2}\left(R^{n}\right)}\|u\|_{L^{2}\left(R^{n}\right)} .
$$

The second equality follows from a similar argument as above and by integrating with respect to $\xi$ first instead of $x$.

Proof OF THEOREM 2. Without loss of generality, we can assume that the symbol $a$ is in $C_{0}^{\infty}\left(R^{n} \times R^{n}\right)$ and its derivatives $\partial_{x}^{\alpha} \partial \xi_{\xi}^{\beta} a$ satisfy (1.3). Then for $u \in C_{0}^{\infty}\left(R^{n}\right)$ and $x \in R^{n}$, we have

$$
a(x, D) u(x)=\left(\frac{1}{2 \pi}\right)^{n} \int_{R^{n}} \int_{R^{n}} e^{i(x-y) \xi} a(x, \xi) u(y) d y d \xi .
$$

Making use of the identity

$$
\left(\prod_{s=1}^{n} \frac{1}{1+i\left(x_{s}-y_{s}\right)}\right)\left(\prod_{s=1}^{n}\left(1+\partial_{\xi_{s}}\right)\right)\left(e^{i(x-y) \xi}\right)=e^{i(x-y) \xi},
$$

we obtain

$$
\int_{R^{n}} e^{i(x-y) \xi} a(x, \xi) d \xi=\int_{R^{n}} e^{i(x-y) \xi} b(x, \xi)\left(\prod_{s=1}^{n} \frac{1}{1+i\left(x_{s}-y_{s}\right)}\right) d \xi
$$

where $b(x, \xi)=\left(\prod_{s=1}^{n}\left(1-\partial_{\xi_{s}}\right)\right)(a(x, \xi))$. Substituting (3.2) into (3.1), we have the following crucial equality:

$$
\begin{aligned}
a(x, D) & u(x) \\
= & \left(\frac{1}{2 \pi}\right)^{n} \int_{R^{n}} \int_{R^{n}} e^{i(x-y) \xi} b(x, \xi)\left(\prod_{s=1}^{n} \frac{1}{1+i\left(x_{s}-y_{s}\right)}\right) u(y) d y d \xi .
\end{aligned}
$$

Let $v \in C_{0}^{\infty}\left(R^{n}\right)$. By (3.3) and

$$
v(x)=\left(\frac{1}{2 \pi}\right)^{n} \int_{R^{n}} e^{i x \lambda} \hat{v}(\lambda) d \lambda,
$$


we have

$$
\begin{aligned}
& \int_{R^{n}} a(x, D) u(x) v(x) d x \\
& =\left(\frac{1}{2 \pi}\right)^{2 n} \int_{R^{n}} \int_{R^{n}} \int_{R^{n}} \int_{R^{n}} e^{i(x-y) \xi} e^{i x \lambda} b(x, \xi) \\
& \times\left(\prod_{s=1}^{n} \frac{1}{1+i\left(x_{s}-y_{s}\right)}\right) u(y) \hat{v}(\lambda) d y d \xi d \lambda d x \\
& =\left(\frac{1}{2 \pi}\right)^{2 n} \int_{R^{n}} \int_{R^{n}} \int_{R^{n}}\left(\int_{R^{n}} \times e^{i x(\xi+\lambda)} b(x, \xi)\right. \\
& \left.\times\left(\prod_{s=1}^{n} \frac{1}{1+i\left(x_{s}-y_{s}\right)}\right) d x\right) e^{-i y \xi} u(y) \hat{v}(\lambda) d y d \xi d \lambda .
\end{aligned}
$$

Integrating with respect to $x$ first and making use of the identity

$$
\left(\prod_{s=1}^{n} \frac{1}{1+i\left(\xi_{s}+\lambda_{s}\right)}\right)\left(\prod_{s=1}^{n}\left(1+\partial_{x}\right)\right)\left(e^{i x(\xi+\lambda)}\right)=e^{i x(\xi+\lambda)},
$$

we get

$$
\begin{aligned}
\int_{R^{n}} e^{i x(\xi+\lambda)} b(x, \xi)\left(\prod_{s=1}^{n} \frac{1}{1+i\left(x_{s}-y_{s}\right)}\right) d x \\
=\left(\prod_{s=1}^{n} \frac{1}{1+i\left(\xi_{s}+\lambda_{s}\right)}\right) \\
\quad \times \int_{R^{n}} e^{i x(\xi+\lambda)}\left(\prod_{s=1}^{n}\left(1-\partial_{x_{s}}\right)\right)\left(b(x, \xi)\left(\prod_{s=1}^{n} \frac{1}{1+i\left(x_{s}-y_{s}\right)}\right)\right) d x .
\end{aligned}
$$

Let $T=\left\{\alpha \in N^{n} \mid \alpha=\left(\alpha_{1}, \ldots, \alpha_{n}\right)\right.$ and $\alpha_{j}=0$ or 1$\}$. Then we have

$$
\begin{aligned}
\left(\prod_{s=1}^{n}\left(1-\partial_{x_{s}}\right)\right)( & \left.b(x, \xi)\left(\prod_{s=1}^{n} \frac{1}{1+i\left(x_{s}-y_{s}\right)}\right)\right) \\
& =\sum_{\alpha \in T}(-1)^{|\alpha|} \partial_{x}^{\alpha}\left(b(x, \xi)\left(\prod_{s=1}^{n} \frac{1}{1+i\left(x_{s}-y_{s}\right)}\right)\right) \\
& =\sum_{\alpha \in T}(-1)^{|\alpha|} \sum_{\beta \leqslant \alpha} \partial_{x}^{\alpha-\beta} b(x, \xi) \partial_{x}^{\beta}\left(\prod_{s=1}^{n} \frac{1}{1+i\left(x_{s}-y_{s}\right)}\right) .
\end{aligned}
$$

Substituting (3.6) into (3.5), we see that

$$
\begin{aligned}
& \int_{R^{n}} e^{i x(\xi+\lambda)} b(x, \xi)\left(\prod_{s=1}^{n} \frac{1}{1+i\left(x_{s}-y_{s}\right)}\right) d x \\
& =\sum_{\alpha \in T} \sum_{\beta \leqslant \alpha}(-1)^{|\alpha|}\left(\prod_{s=1}^{n} \frac{1}{1+i\left(\xi_{s}+\lambda_{s}\right)}\right) \\
& \times \int_{R^{n}} e^{i x(\xi+\lambda)} \partial_{x}^{\alpha-\beta} b(x, \xi) \partial_{x}^{\beta}\left(\prod_{s=1}^{n} \frac{1}{1+i\left(x_{s}-y_{s}\right)}\right) d x
\end{aligned}
$$


We derive from (3.4) and (3.7) that

$$
\begin{array}{rl}
\int_{R^{n}} & a(x, D) u(x) v(x) d x \\
& =\sum_{\alpha \in T} \sum_{\beta \leqslant \alpha}(-1)^{|\alpha|}\left(\frac{1}{2 \pi}\right)^{2 n} \int_{R^{n}} \int_{R^{n}} \partial_{x}^{\alpha-\beta} b(x, \xi) g_{\beta}(x, \xi) h(x, \xi) d x d \xi
\end{array}
$$

where

$$
g_{\beta}(x, \xi)=\int_{R^{n}} e^{i(x-y) \xi} \partial_{x}^{\beta}\left(\prod_{s=1}^{n} \frac{1}{1+i\left(x_{s}-y_{s}\right)}\right) u(y) d y
$$

and

$$
h(x, \xi)=\int_{R^{n}} e^{i x \lambda}\left(\prod_{s=1}^{n} \frac{1}{1+i\left(\xi_{s}+\lambda_{s}\right)}\right) \hat{v}(\lambda) d \lambda .
$$

Applying Lemma (3.1) to $g_{\beta}$, we get

$$
\begin{aligned}
\left\|g_{\beta}\right\|_{L^{2}\left(R^{n} \times R^{n}\right)} & =(2 \pi)^{n / 2}\left(\int_{R^{n}}\left|\partial_{x}^{\beta}\left(\prod_{s=1}^{n} \frac{1}{1+i x_{s}}\right)\right|^{2} d x\right)^{1 / 2}\|u\|_{L^{2}\left(R^{n}\right)} \\
& \leqslant(2 \pi)^{n / 2} \pi^{n / 2}\|u\|_{L^{2}\left(R^{n}\right) .}
\end{aligned}
$$

Similarly, we have

$$
\begin{aligned}
\|h\|_{L^{2}\left(R^{n} \times R^{n}\right)} & =(2 \pi)^{n / 2}\left(\int_{R^{n}} \prod_{s=1}^{n} \frac{1}{1+\xi_{s}^{2}} d \xi\right)^{1 / 2}\|\hat{v}\|_{L^{2}\left(R^{n}\right)} \\
& =(2 \pi)^{n} \pi^{n / 2}\|v\|_{L^{2}\left(R^{n}\right)}
\end{aligned}
$$

From (1.3), we see that

$$
\left\|\partial_{x}^{\alpha-\beta_{b}}\right\|_{L^{\infty}\left(R^{n} \times R^{n}\right)} \leqslant 2^{n}\|\mid a\| .
$$

Thus, we derive from (3.8), (3.9), (3.10) and (3.11) that

$$
\left|\int_{R^{n}} a(x, D) u(x) v(x) d x\right| \leqslant C_{n}\|\mid a\|\|u\|_{L^{2}\left(R^{n}\right)}\|v\|_{L^{2}\left(R^{n}\right)},
$$

where $u, v \in C_{0}^{\infty}\left(R^{n}\right)$ and $C_{n}$ is a constant depending only on $n$.

Proof of COROLlary 2.1. Without loss of generality, we can assume that the symbol $a$ has compact support in $R^{n} \times R^{n}$ and that its derivatives $\partial \xi a$ satisfy (1.4). Then we approach the symbol $a$ by the smooth symbols $a_{\varepsilon}$ defined by $a_{\varepsilon}=\lambda_{\varepsilon} * a$, where $0<\varepsilon<1$ and $\lambda_{\varepsilon}$ is as in (2.13). By the definition of $a_{\varepsilon}$ and taking into account that $a \in L^{2}\left(R^{n} \times R^{n}\right)$, we have the following properties:

$$
\begin{gathered}
a_{\varepsilon} \in C_{0}^{\infty}\left(R^{n} \times R^{n}\right), \\
\lim _{\varepsilon \rightarrow 0}\left\|a_{\varepsilon}-a\right\|_{L^{2}\left(R^{n} \times R^{n}\right)}=0
\end{gathered}
$$


and

(3.14) $\sup _{x \in R^{n}}\left(\int\left|\partial_{\xi}^{\beta} a_{\varepsilon}(x, \xi)\right|^{2} d \xi\right)^{1 / 2} \leqslant\|\| a \|, \quad \beta=\left(\beta_{1}, \ldots, \beta_{n}\right), \beta_{j}=0$ or 1 .

Let $u \in C_{0}^{\infty}\left(R^{n}\right)$ and $x \in R^{n}$. From (3.3), we have (3.15) $a_{\varepsilon}(x, D) u(x)$

$$
=\left(\frac{1}{2 \pi}\right)^{n} \int_{R^{n}} \int_{R^{n}} e^{i(x-y) \xi} b_{\varepsilon}(x, \xi)\left(\prod_{s=1}^{n} \frac{1}{1+i\left(x_{s}-y_{s}\right)}\right) u(y) d y d \xi,
$$

where

$$
b_{\varepsilon}(x, \xi)=\left(\prod_{s=1}^{n}\left(1-\partial_{\xi_{s}}\right)\right)\left(a_{\varepsilon}(x, \xi)\right) .
$$

Let $v \in C_{0}^{\infty}\left(R^{n}\right)$. Making use of (3.15), we write

$$
\begin{aligned}
& \int_{R^{n}} a_{\varepsilon}(x, D) u(x) v(x) d x \\
&=\left(\frac{1}{2 \pi}\right)^{n} \int_{R^{n}} \int_{R^{n}} b_{\varepsilon}(x, \xi) v(x) \\
& \times\left(\int_{R^{n}} e^{i(x-y) \xi}\left(\prod_{s=1}^{n} \frac{1}{1+i\left(x_{s}-y_{s}\right)}\right) u(y) d y\right) d x d \xi \\
&=\left(\frac{1}{2 \pi}\right)^{n} \int_{R^{n}} \int_{R^{n}} g(x, \xi) h(x, \xi) d x d \xi,
\end{aligned}
$$

where $g(x, \xi)=b_{\varepsilon}(x, \xi) v(x)$, and

$$
h(x, \xi)=\int_{R^{n}} e^{i(x-y) \xi}\left(\prod_{s=1}^{n} \frac{1}{1+i\left(x_{s}-y_{s}\right)}\right) u(y) d y .
$$

By (3.14) and

$$
b_{\varepsilon}(x, \xi)=\left(\prod_{s=1}^{n}\left(1-\partial_{\xi_{s}}\right)\right)\left(a_{\varepsilon}(x, \xi)\right)
$$

we see that

$$
\|g\|_{L^{2}\left(R^{n} \times R^{n}\right)} \leqslant 2^{n}\|\| a\|\|\|v\|_{L^{2}\left(R^{n}\right) .}
$$

According to Lemma (3.1), we have

$$
\begin{aligned}
\|h\|_{L^{2}\left(R^{n} \times R^{n}\right)} & =(2 \pi)^{n / 2}\left(\int_{R^{n}} \prod_{s=1}^{n} \frac{1}{1+x_{s}^{2}} d x\right)^{1 / 2}\|u\|_{L^{2}\left(R^{n}\right)} \\
& \leqslant(2 \pi)^{n}\|u\|_{L^{2}\left(R^{n}\right) .}
\end{aligned}
$$

Therefore, we derive from (3.16), (3.17) and (3.18) that

(3.19) $\left|\int_{R^{n}} a_{\varepsilon}(x, D) u(x) v(x) d x\right| \leqslant 2^{n}\|a\|\|\| u\left\|_{L^{2}\left(R^{n}\right)}\right\| v \|_{L^{2}\left(R^{n}\right)}$

$$
\forall u, v \in C_{0}^{\infty}\left(R^{n}\right)
$$


Now, we are ready to prove the $L^{2}$-boundedness of $a(x, D)$. For $u, v \in C_{0}^{\infty}\left(R^{n}\right)$, we write

$$
\int_{R^{n}} a_{\varepsilon}(x, D) u(x) v(x) d x=\left(\frac{1}{2 \pi}\right)^{n} \int_{R^{n}} \int_{R^{n}} e^{i x \xi} a_{\varepsilon}(x, \xi) \hat{u}(\xi) v(x) d x d \xi .
$$

Making use of (3.13), we see that

$$
\begin{aligned}
& \lim _{\varepsilon \rightarrow 0} \int_{R^{n}} \int_{R^{n}} e^{i x \xi} a_{\varepsilon}(x, \xi) \hat{u}(\xi) v(x) d x d \xi \\
& \quad=\int_{R^{n}} \int_{R^{n}} e^{i x \xi} a(x, \xi) \hat{u}(\xi) v(x) d x d \xi, \quad u, v \in C_{0}^{\infty}\left(R^{n}\right) .
\end{aligned}
$$

Therefore we derive from (3.19) and (3.20) that

$$
\left|\int_{R^{n}} a(x, D) u(x) v(x) d x\right| \leqslant 2^{n}\|a\|\|\| u\left\|_{L^{2}\left(R^{n}\right)}\right\| v \|_{L^{2}\left(R^{n}\right)} \quad \forall u, v \in C_{0}^{\infty}\left(R^{n}\right) .
$$

Thus

$$
\|a(x, D) u\|_{L^{2}\left(R^{n}\right)} \leqslant 2^{n}\|a\|\|\| u \|_{L^{2}\left(R^{n}\right),} \quad u \in C_{0}^{\infty}\left(R^{n}\right) .
$$

Proof of Corollary 2.2. Without loss of generality, we can assume that the symbol $a \in C_{0}^{\infty}\left(R^{n} \times R^{n}\right)$ and its derivatives $\partial_{x}^{\alpha} a$ satisfy (1.5). If $u, v \in C_{0}^{\infty}\left(R^{n}\right)$, we have

$$
\begin{aligned}
\int_{R^{n}} a(x, D) u(x) v(x) d x & =\left(\frac{1}{2 \pi}\right)^{n} \int_{R^{n}}\left(\int_{R^{n}} e^{i x \xi} a(x, \xi) \hat{u}(\xi) d \xi\right) v(x) d x \\
& =\left(\frac{1}{2 \pi}\right)^{n} \int_{R^{n}}\left(\int_{R^{n}} e^{i x \xi} a(x, \xi) v(x) d x\right) \hat{u}(\xi) d \xi \\
& =\int_{R^{n}} g(\xi) \hat{u}(\xi) d \xi
\end{aligned}
$$

where

$$
g(\xi)=\left(\frac{1}{2 \pi}\right)^{n} \int_{R^{n}} e^{i x \xi} a(x, \xi) v(x) d x .
$$

It follows from Corollary (2.1) that

$$
\|g\|_{L^{2}\left(R^{n}\right)} \leqslant(2 \pi)^{-n} 2^{n}\|a\|\|\| \hat{v}\left\|_{L^{2}\left(R^{n}\right)}=(2 \pi)^{-n / 2} 2^{n}\right\| a\|\|\|v\|_{L^{2}\left(R^{n}\right) .}
$$

From (3.21) and (3.22), we have

$$
\left|\int_{R^{n}} a(x, D) u(x) v(x) d x\right| \leqslant\|g\|_{L^{2}\left(R^{n}\right)}\|\hat{u}\|_{L^{2}\left(R^{n}\right)} \leqslant 2^{n}\|a\|\|\| u\left\|_{L^{2}\left(R^{n}\right)}\right\| v \|_{L^{2}\left(R^{n}\right)}
$$

Thus

$$
\|a(x, D) u\|_{L^{2}\left(R^{n}\right)} \leqslant 2^{n}\|a\|\|\| u \|_{L^{2}\left(R^{n}\right),} \quad u \in C_{0}^{\infty}\left(R^{n}\right) .
$$

4. Proof of Theorem 3. The case $\rho=0$ has been proved in Theorem 2. It remains to prove the theorem for the cases $-\infty<\rho<1, \rho \neq 0$. Throughout this section, we denote a constant depending only on $\rho$ and $n$ by $C_{\rho, n}$, which may vary from time to time. We may assume that $a$ is in $C_{0}^{\infty}\left(R^{n} \times R^{n}\right)$ and its derivatives $\partial_{x}^{\alpha} \partial \xi \beta a$ satisfy 
(1.6) for some $-\infty<\rho<1, \rho \neq 0$. Making use of the partition of unity in [2] i.e. there exist $\tilde{\varphi}_{0}, \varphi \in C_{0}^{\infty}\left(R^{n}\right)$ such that $\operatorname{supp} \varphi \subseteq\left\{\frac{1}{3} \leqslant|\xi| \leqslant 1\right\} \quad$ and $\quad \tilde{\varphi}_{0}+\sum_{j=0}^{\infty} \varphi_{j} \equiv 1 \quad$ with $\varphi_{j}(\xi)=\varphi\left(2^{-j} \xi\right), \xi \in R^{n}$, we write

$$
\begin{aligned}
a(x, \xi) & =\tilde{\varphi}_{0}(\xi) a(x, \xi)+\sum_{j=0}^{\infty} \varphi_{j}(\xi) a(x, \xi) \\
& =b_{0}(x, \xi)+\sum_{j=0}^{\infty} a_{j}(x, \xi), \quad(x, \xi) \in R^{n} \times R^{n} .
\end{aligned}
$$

Then

$$
a(x, D) u=b_{0}(x, D) u+\sum_{j=0}^{\infty} a_{j}(x, D) u, \quad u \in C_{0}^{\infty}\left(R^{n}\right) .
$$

It follows from Corollary 2.1 that $b_{0}(x, D)$ and $a_{0}(x, D)$ are continuous from $L^{2}\left(R^{n}\right)$ to $L^{2}\left(R^{n}\right)$ with their norms bounded by $C_{\rho, n}\|a\|$. Therefore we shall concentrate on estimating $\sum_{j=0}^{\infty} a_{j}(x, D) u$. Since the symbol $a$ is in $C_{0}^{\infty}\left(R^{n} \times R^{n}\right)$, we obtain

$\left(\sum_{j=1}^{\infty} a_{j}(x, D) u, \sum_{j=1}^{\infty} a_{j}(x, D) u\right)=\sum_{j, k=1}^{\infty}\left(a_{j}(x, D) u, a_{k}(x, D) u\right)$,

$$
u \in C_{0}^{\infty}\left(R^{n}\right) .
$$

We shall give several lemmas to study the behavior of $\left(a_{j}(x, D) u, a_{k}(x, D) u\right)$. Then we shall see that Theorem 3 follows from these lemmas.

Lemma 4.1. Let $j \in N-\{0\}$ and $E_{j}=\left\{\xi \in R^{n}\left|2^{j} / 3 \leqslant\right| \xi \mid \leqslant 2^{j}\right\}$. Then

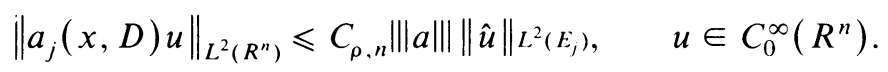

Proof. Since supp $a(x, \cdot) \subseteq E_{j}$ and

$$
a_{j}(x, D) u(x)=\left(\frac{1}{2 \pi}\right)^{n} \int_{R^{n}} e^{i x \xi} a(x, \xi) \hat{u}(\xi) d \xi, \quad x \in R^{n}, u \in C_{0}^{\infty}\left(R^{n}\right),
$$

it suffices to show the following property:

$$
\left\|a_{j}(x, D) u\right\|_{L^{2}\left(R^{n}\right)} \leqslant C_{\rho, n}\|a\|\|\| \hat{u} \|_{L^{2}\left(E_{j}\right)}, \quad \hat{u} \in C_{0}^{\infty}\left(E_{j}\right) .
$$

Let $v \in C_{0}^{\infty}\left(R^{n}\right)$. Then

$$
\begin{aligned}
\int_{R^{n}} a_{j}(x, D) u(x) v(x) d x & =\left(\frac{1}{2 \pi}\right)^{n} \int_{R^{n}} \int_{R^{n}} e^{i x \xi} a_{j}(x, \xi) \hat{u}(\xi) v(x) d \xi d x \\
= & \left(\frac{1}{2 \pi}\right)^{n} \int_{R^{n}} \int_{R^{n}} e^{i x \xi} a_{j}\left(2^{-j \rho} x, 2^{j \rho} \xi\right) \hat{u}\left(2^{j \rho} \xi\right) v\left(2^{-j \rho} x\right) d \xi d x,
\end{aligned}
$$

where the last equality is obtained by the change of variables $x \rightarrow 2^{-j \rho} x, \xi \rightarrow 2^{j \rho} \xi$. By the hypothesis (1.6), we have

$$
\left|\partial_{x}^{\alpha} \partial \xi\left(a_{j}\left(2^{-j \rho} x, 2^{j \rho} \xi\right)\right) \leqslant C_{\rho, n}\||| a|\||,\right.
$$

where $(x, \xi) \in R^{n} \times R^{n}, j \geqslant 1$, and $\alpha=\left(\alpha_{1}, \ldots, \alpha_{n}\right), \beta=\left(\beta_{1}, \ldots, \beta_{n}\right) \in N^{n}$ with 
$\alpha_{t}=0$ or 1 and $\beta_{t}=0$ or 1 . So by the proof of Theorem 2 , we have

$$
\left|\int_{R^{n}} a_{j}(x, D) u(x) v(x) d x\right| \leqslant C_{\rho, n}\|a\|\|\| \hat{u}\left\|_{L^{2}\left(E_{j}\right)}\right\| v \|_{L^{2}\left(R^{n}\right)},
$$

which implies (4.1).

Lemma 4.1 implies the following lemma for which we omit the proof.

Lemma 4.2. If $s \in N-\{0\}$ and $j, k \geqslant 1$, then

$$
\left.\sum_{\forall-k \mid \leqslant s} \mid a_{j}(x, D) u, a_{k}(x, D) u\right) \mid \leqslant s^{2} C_{\rho, n}\|a\|^{2}\|u\|_{L^{2}\left(R^{n}\right)}^{2} \quad \forall u \in C_{0}^{\infty}\left(R^{n}\right) .
$$

The following lemmas describe the properties of $\left(a_{j}(x, D) u, a_{k}(x, D) u\right)$, when $|j-k|$ is large.

LEMMA 4.3. If $0<\rho<1$, then we have

$$
\left|\left(a_{j}(x, D) u, a_{k}(x, D) v\right)\right| \leqslant C_{\rho, n}\left\|\left|\|\mid\|^{2} 2^{-j \varepsilon_{\rho} 2^{-k \varepsilon_{\rho}}\|\hat{u}\|}\left\|_{L^{2}\left(E_{j}\right)}\right\| \hat{v} \|_{L^{2}\left(E_{k}\right)},\right.\right.
$$

where $u, v \in C_{0}^{\infty}\left(R^{n}\right), j, k \geqslant 1 ;|j-k| \geqslant 4$ and $\varepsilon_{\rho}=(1-\rho) / 4$.

Proof. Since supp $a_{j}(x, \cdot)$ and supp $a_{k}(x, \cdot)$ are contained in $E_{j}$ and $E_{k}$ respectively, it suffices to show the following property:

$$
\left|\left(a_{j}(x, D) u, a_{k}(x, D) v\right)\right| \leqslant C_{\rho, n}\|\mid a\|^{2} 2^{-j \varepsilon_{\rho}} 2^{-k \varepsilon_{\rho}}\|\hat{u}\| L^{2}\left(E_{j}\right)\|\hat{v}\|_{L^{2}\left(E_{k}\right)},
$$

where $\hat{u} \in C_{0}^{\infty}\left(E_{j}\right), \hat{v} \in C_{0}^{\infty}\left(E_{k}\right), \quad j, \quad k \geqslant 1, \quad|j-k| \geqslant 4$ and $\varepsilon_{\rho}=(1-\rho) / 4$. Without loss of generality, we can assume that $j \geqslant k+4$. Then we write

$$
\begin{aligned}
&\left(a_{j}(x, D) u, a_{k}(x, D) v\right) \\
&=\left(\frac{1}{2 \pi}\right)^{2 n} \int_{R^{n}} \int_{R^{n}} \int_{R^{n}} e^{i x(\xi-\lambda)} a_{j}(x, \xi) \hat{u}(\xi) \bar{a}_{k}(x, \lambda) \overline{\hat{v}}(\lambda) d \lambda d \xi d x \\
&=\left(\frac{1}{2 \pi}\right)^{2 n} 2^{j \rho n} \iiint e^{i x(\xi-\lambda)} a_{j}\left(2^{-j \rho} x, 2^{j \rho} \xi\right) \hat{u}\left(2^{j \rho} \xi\right) \\
& \times \bar{a}_{k}\left(2^{-j \rho} x, 2^{j \rho} \xi\right) \overline{\hat{v}}\left(2^{j \rho} \lambda\right) d \lambda d \xi d x,
\end{aligned}
$$

where the last equality comes from the change of variables $x \rightarrow 2^{-j \rho} x, \xi \rightarrow 2^{j \rho} \xi$ and $\lambda \rightarrow 2^{j \rho} \lambda$.

In the last integral, we integrate with respect to $x$ first. By using the following identity:

$$
\left(\prod_{s=1}^{n} \frac{1}{1+i\left(\xi_{s}-\lambda_{s}\right)}\right)\left(\prod_{s=1}^{n}\left(1+\partial_{x_{s}}\right)\right)\left(e^{i x(\xi-\lambda)}\right)=e^{i x(\xi-\lambda)},
$$

we write

$$
\begin{aligned}
\int_{R^{n}} e^{i x(\xi-\lambda)} a_{j}\left(2^{-j \rho} x, 2^{j \rho} \xi\right) \bar{a}_{k}\left(2^{-j \rho} x, 2^{j \rho} \lambda\right) d x \\
=\left(\prod_{s=1}^{n} \frac{1}{1+i\left(\xi_{s}-\lambda_{s}\right)}\right) \\
\quad \times \sum_{\alpha \in T} \sum_{\beta \leqslant \alpha}(-1)^{|\alpha|} \int_{R^{n}} e^{i x(\xi-\lambda)} \partial_{x}^{\alpha-\beta}\left(a_{j}\left(2^{-j \rho} x, 2^{j \rho} \xi\right)\right) \partial_{x}^{\beta}\left(a_{k}\left(2^{-j \rho} x, 2^{j \rho} \lambda\right)\right) d x,
\end{aligned}
$$


where $T=\left\{\alpha \in N^{n} \mid \alpha=\left(\alpha_{1}, \ldots, \alpha_{n}\right)\right.$ with $\alpha_{t}=0$ or 1$\}$. Substituting (4.4) into (4.3), we obtain

$$
\left(a_{j}(x, D) u, a_{k}(x, D) v\right)=\left(\frac{1}{2 \pi}\right)^{2 n} 2^{j \rho n} \sum_{\alpha \in T} \sum_{\beta \leqslant \alpha}(-1)^{|\alpha|} I_{\alpha, \beta},
$$

where

$$
\begin{aligned}
I_{\alpha, \beta}=\int_{R^{n}} \int_{R^{n}} \int_{R^{n}} e^{i x(\xi-\lambda)} \partial_{x}^{\alpha-\beta}\left(a_{j}\left(2^{-j \rho} x, 2^{j \rho} \xi\right)\right) \partial_{x}^{\beta}\left(\bar{a}_{k}\left(2^{-j \rho} x, 2^{j \rho} \lambda\right)\right) \\
\quad \times\left(\prod_{s=1}^{n} \frac{1}{1+i\left(\xi_{s}-\lambda_{s}\right)}\right) \hat{u}\left(2^{j \rho} \xi\right) \overline{\hat{v}}\left(2^{j \rho} \lambda\right) d \lambda d \xi d x
\end{aligned}
$$

Let $\hat{g}(\xi)=\hat{u}\left(2^{j \rho} \xi\right), \xi \in R^{n}$. Then

$$
\hat{g}(\xi)=\int_{R^{n}} e^{-i y \xi} g(y) d y .
$$

Substituting (4.7) into (4.6), integrating with respect to $\xi$ first and making use of the following identity:

$$
\left(\prod_{s=1}^{n} \frac{1}{1+i\left(x_{s}-y_{s}\right)}\right)\left(\prod_{s=1}^{n}\left(1+\partial_{\xi_{s}}\right)\right)\left(e^{i(x-y) \xi}\right)=e^{i(x-y) \xi},
$$

We obtain

$$
I_{\alpha, \beta}=\sum_{\gamma \in T} \sum_{\delta<\gamma}(-1)^{|\gamma|} J_{\alpha, \beta, \gamma, \delta},
$$

where

$$
\text { (4.9) } \begin{aligned}
J_{\alpha, \beta, \gamma, \delta}= & \int_{R^{n}} \int_{R^{n}} \int_{R^{n}} \int_{R^{n}} e^{i(x-y) \xi} \partial_{\xi}^{\gamma-\delta} \partial_{x}^{\alpha-\beta}\left(a_{j}\left(2^{-j \rho} x, 2^{j \rho} \xi\right)\right) \\
& \times \partial_{\xi}^{\delta}\left(\prod_{s=1}^{n} \frac{1}{1+i\left(\xi_{s}-\lambda_{s}\right)}\right)\left(\prod_{s=1}^{n} \frac{1}{1+i\left(x_{s}-y_{s}\right)}\right) g(y) e^{-i x \lambda} \\
& \times \partial_{x}^{\beta}\left(\bar{a}_{k}\left(2^{-j \rho} x, 2^{j \rho} \lambda\right)\right) \overline{\hat{v}}\left(2^{j \rho} \lambda\right) d y d \lambda d \xi d x \\
= & \int_{R^{n}} \int_{R^{n}} \partial_{\xi}^{\gamma-\delta} \partial_{x}^{\alpha-\beta}\left(a_{j}\left(2^{-j \rho} x, 2^{j \rho} \xi\right)\right) G(x, \xi) H_{\beta, \delta}(x, \xi) d x d \xi,
\end{aligned}
$$

with

$$
G(x, \xi)=\int_{R^{n}} e^{i(x-y) \xi}\left(\prod_{s=1}^{n} \frac{1}{1+i\left(x_{s}-y_{s}\right)}\right) g(y) d y,
$$

and

$$
\begin{aligned}
H_{\beta, \delta}(x, \xi)=\int_{R^{n}} e^{-i x \lambda} \partial_{x}^{\beta}\left(\overline { a } _ { k } \left(2^{-j \rho} x\right.\right. & \left.\left., 2^{j \rho} \lambda\right)\right) \\
& \times \partial_{\xi}^{\beta}\left(\prod_{s=1}^{n} \frac{1}{1+i\left(\xi_{s}-\lambda_{s}\right)}\right) \bar{v}\left(2^{j \rho} \lambda\right) d \lambda .
\end{aligned}
$$


By (4.5) and (4.8) we have

$$
\left(a_{j}(x, D) u, a_{k}(x, D) v\right)=\left(\frac{1}{2 \pi}\right)^{2 n} 2^{j \rho n} \sum_{\alpha \in T} \sum_{\gamma \in T} \sum_{\beta \leqslant \alpha} \sum_{\delta \leqslant \gamma}(-1)^{|\alpha|+|\gamma|} J_{\alpha, \beta, \gamma, \delta} .
$$

Therefore, (4.2) follows by showing that

$$
\left|J_{\alpha, \beta, \gamma, \delta}\right| \leqslant 2^{-j \rho n} C_{\rho, n}\|a\|^{2} 2^{-j \varepsilon_{\rho}} 2^{-k \varepsilon_{\rho}}\|\hat{u}\| L_{L^{2}\left(E_{j}\right)}\|\hat{v}\|_{L^{2}\left(E_{k}\right)},
$$

where $\alpha, \gamma \in T, \beta \leqslant \alpha, \delta \leqslant \gamma$ and $\varepsilon_{\rho}=(1-\rho) / 4$. By Lemma 3.1 and (4.7), we get

$$
\|G\|_{L^{2}\left(R^{n} \times R^{n}\right)}=\pi^{n / 2} 2^{-j \rho n / 2}\|\hat{u}\|_{L^{2}\left(E_{j}\right)} .
$$

By the hypothesis (1.6), we have

$$
\begin{aligned}
\left|\partial_{\xi}^{\gamma-\delta_{\xi}} \partial_{x}^{\alpha-\beta}\left(a_{j}\left(2^{-j \rho} x, 2^{j \rho} \xi\right)\right)\right| & \leqslant C_{\rho, n}\|a \mid\| \\
\text { for }(x, \xi) & \in R^{n} \times R^{n}, \alpha, \beta, \gamma, \delta \in T \text { and } \beta \leqslant \alpha, \delta \leqslant \gamma .
\end{aligned}
$$

It remains to estimate $\left\|H_{\beta, \delta}\right\|_{L^{2}\left(R^{n} \times R^{n}\right)}$. (4.11) can be written as

$$
\begin{aligned}
H_{\beta, \delta}(x, \xi) & =\int_{R^{n}} e^{-i x \lambda} 2^{-j \rho|\beta|}\left(\partial_{x}^{\beta} \bar{a}_{k}\right)\left(2^{-j \rho} x, 2^{j \rho} \lambda\right) \\
& \times \partial_{\xi}^{\delta}\left(\prod_{s=1}^{n} \frac{1}{1+i\left(\xi_{s}-\lambda_{s}\right)}\right) \overline{\hat{v}}\left(2^{j \rho} \lambda\right) d \lambda \\
& =\int_{R^{n}} e^{-i x \lambda} 2^{-j \rho|\beta|}\left(\partial_{x}^{\beta} \bar{a}_{k}\right)\left(2^{-j \rho} x, 2^{j \rho} \lambda\right) h_{\xi}(\lambda) d \lambda,
\end{aligned}
$$

where

$$
h_{\xi}(\lambda)=\partial_{\xi}^{\delta}\left(\prod_{s=1}^{n} \frac{1}{1+i\left(\xi_{s}-\lambda_{s}\right)}\right) \overline{\hat{v}}\left(2^{j \rho} \lambda\right) .
$$

We first estimate $\left\|H_{\beta, \delta}(\cdot, \xi)\right\|_{L^{2}\left(R^{n}\right)}$, so let $w \in C_{0}^{\infty}\left(R^{n}\right)$, then

$$
\begin{aligned}
\int_{R^{n}} H_{\beta, \delta} & (x, \xi) w(x) d x \\
= & \int_{R^{n}} \int_{R^{n}} e^{-i x \lambda} 2^{-j \rho|\beta|}\left(\partial_{x}^{\beta} \bar{a}_{k}\right)\left(2^{-j \rho} x, 2^{j \rho} \lambda\right) h_{\xi}(\lambda) \cdot w(x) d \lambda d x \\
= & \int_{R^{n}} \int_{R^{n}} e^{-i x \lambda} 2^{-j \rho|\beta|}\left(\partial_{x}^{\beta} \bar{a}_{k}\right)\left(2^{-k \rho} x, 2^{k \rho} \lambda\right) \\
& \times h_{\xi}\left(2^{(-j+k) \rho} \lambda\right) w\left(2^{(j-k) \rho} x\right) d \lambda d x .
\end{aligned}
$$

By (1.6) and by the assumption that $j \geqslant k+4$, we have

$$
2^{-j \rho|\beta|}\left|\partial_{x}^{\alpha^{\prime}} \partial_{\xi}^{\beta^{\prime}}\left(\left(\partial_{x}^{\beta} \bar{a}_{k}\right)\left(2^{-k \rho} x, 2^{k \rho} \lambda\right)\right)\right| \leqslant C_{\rho, n}\|a\| \|,
$$

where $(x, \lambda) \in R^{n} \times R^{n}$ and $\alpha^{\prime}, \beta^{\prime}, \beta \in T$.

Thus, we derive from Theorem 2 that

$$
\left|\int_{R^{n}} H_{\beta, \delta}(x, \xi) w(x) d x\right| \leqslant C_{\rho, n}\|a\|\|\| h_{\xi}(\cdot)\left\|_{L^{2}\left(R^{n}\right)}\right\| w \|_{L^{2}\left(R^{n}\right),} \quad w \in C_{0}^{\infty}\left(R^{n}\right),
$$


which implies

$$
\int_{R^{n}}\left|H_{\beta, \delta}(x, \xi)\right|^{2} d x \leqslant C_{\rho, n}\left\|\left.|| a\left|\|^{2} \int_{R^{n}}\right| \partial_{\xi}^{\delta}\left(\prod_{s=1}^{n} \frac{1}{1+i\left(\xi_{s}-\lambda_{s}\right)}\right)\right|^{2}\left|\hat{v}\left(2^{j \rho} \lambda\right)\right|^{2} d \lambda .\right.
$$

Then we have

$$
\begin{aligned}
& \int_{2^{-j \rho} E_{j}}\left(\int_{R^{n}}\left|H_{\beta, \delta}(x, \xi)\right|^{2} d x\right) d \xi \\
& \quad \leqslant C_{\rho, n}\||| a \mid\|^{2} \int_{2^{-j \rho} E_{j}}\left(\int_{R^{n}}\left|\partial_{\xi}^{\delta}\left(\prod_{s=1}^{n} \frac{1}{1+i\left(\xi_{s}-\lambda_{s}\right)}\right)\right|^{2}\left|\hat{v}\left(2^{j \rho} \lambda\right)\right|^{2} d \lambda\right) d \xi
\end{aligned}
$$

Since $\delta=\left(\delta_{1}, \ldots, \delta_{n}\right) \in T$, we see that

$$
\left|\partial_{\xi}^{\delta}\left(\prod_{s=1}^{n} \frac{1}{1+i\left(\xi_{s}-\lambda_{s}\right)}\right)\right|^{2} \leqslant \prod_{s=1}^{n} \frac{1}{1+\left(\xi_{s}-\lambda_{s}\right)^{2}}, \quad \lambda, \xi \in R^{n} .
$$

Then an elementary calculation gives

$$
\int_{2^{-j \rho} E_{j}} \prod_{s=1}^{n} \frac{1}{1+\left(\xi_{s}-\lambda_{s}\right)^{2}} d \xi \leqslant 2^{8} n^{2} \pi^{n} 2^{-j(1-\rho)}
$$

$$
\text { for } \lambda \in 2^{-j \rho} E_{k} \text { and } j \geqslant k+4 .
$$

Thus, it follows from (4.15), (4.16), (4.17) and Fubini's theorem that

$$
\left(\int_{2^{-j \rho} E_{j}} \int_{R^{n}}\left|H_{\beta, \delta}(x, \xi)\right|^{2} d x d \xi\right)^{1 / 2} \leqslant C_{\rho, n}\|a\| 2^{-j(1-\rho) / 2} 2^{-j \rho n / 2}\|\hat{v}\| L^{2}\left(E_{k}\right) .
$$

By (4.9), and the fact that $\operatorname{supp} a_{j}(x, \cdot) \subseteq E_{j}$, we obtain

$$
\left|J_{\alpha, \beta, \gamma, \delta}\right| \leqslant C_{\rho, n}\|a\|\|\| G\left\|L_{L^{2}\left(R^{n} \times 2^{-j \rho} E_{j}\right)}\right\| H \|_{L^{2}}\left(R^{n} \times 2^{-j \rho} E_{j}\right) .
$$

Thus (4.12) follows from (4.13), (4.18) and (4.19).

LEMMA 4.4. If $-\infty<\rho<0$, then

$$
\left|\left(a_{j}(x, D) u, a_{k}(x, D) v\right)\right| \leqslant C_{\rho, n}\|a\|^{2} 2^{-j / 4} 2^{-k / 4}\|\hat{u}\| L^{2}\left(E_{j}\right)\|\hat{v}\|_{L^{2}\left(E_{k}\right)},
$$

where $u, v \in C_{0}^{\infty}\left(R^{n}\right), j, k \geqslant 1$ and $|j-k| \geqslant 4$.

Proof. Without loss of generality, we can assume that $\hat{u} \in C_{0}^{\infty}\left(E_{j}\right), \hat{v} \in C_{0}^{\infty}\left(E_{k}\right)$ and $j \geqslant k+4$. Then

$$
\begin{aligned}
&\left(a_{j}(x, D) u, a_{k}(x, D) v\right) \\
&=\left(\frac{1}{2 \pi}\right)^{2 n} \int_{R^{n}} \int_{R^{n}} \int_{R^{4}} e^{i x(\xi-\lambda)} a_{j}(x, \xi) \hat{u}(\xi) \bar{a}_{k}(x, \lambda) \hat{\hat{v}}(\lambda) d \lambda d \xi d x \\
&=\left(\frac{1}{2 \pi}\right)^{2 n} 2^{k \rho n} \int_{R^{n}} \int_{R^{n}} \int_{R^{n}} e^{i x(\xi-\lambda)} a_{j}\left(2^{-k \rho} x, 2^{k \rho} \xi\right) \hat{u}\left(2^{k \rho} \xi\right) \\
& \quad \times \bar{a}_{k}\left(2^{-k \rho} x, 2^{k \rho} \lambda\right) \overline{\hat{v}}\left(2^{k \rho} \lambda\right) d \lambda d \xi d x
\end{aligned}
$$


where the last equality comes from change of variables $x \rightarrow 2^{-k \rho} x, \xi \rightarrow 2^{k \rho} \xi$, $\lambda \rightarrow 2^{k \rho} \lambda$. We will use a technique similar to the proof of Lemma 4.3 , but we integrate with respect to $\lambda$ instead of integrating with respect to $\xi$. Then the lemma follows by using the inequality

$$
\int_{2^{-k \rho} E_{k}} \prod_{s=1}^{n} \frac{1}{1+\left|\xi_{s}-\lambda_{s}\right|^{2}} d \lambda \leqslant 2^{8} n^{2} \pi^{n} \cdot 2^{-j+k \rho} \text { for } \xi \in 2^{-k \rho} E_{j} .
$$

Thus, Theorem 3 follows from Lemmas 4.1, 4.2, 4.3, and 4.4.

5. Proof of Theorem 4. By using the same partition of unity as in $\S 4$, we have

$$
a(x, D) u(x)=b_{0}(x, D) u(x)+\sum_{j=0}^{\infty} a_{j}(x, D) u(x), \quad \text { for } u \in C_{0}^{\infty}\left(R^{n}\right) .
$$

By Corollary 2.1, it suffices to estimate $\left\|\sum_{j=5}^{\infty} a_{j}(x, D) u\right\|_{L^{2}\left(R^{n}\right)}$ with $u \in C_{0}^{\infty}\left(R^{n}\right)$. We write

$$
\left\|\sum_{j=5}^{\infty} a_{j}(x, D) u\right\|_{L^{2}\left(R^{n}\right)}^{2}=\sum_{j, k=5}^{\infty}\left(a_{j}(x, D) u, a_{k}(x, D) u\right) .
$$

The only difficulty in estimating $\left|\left(a_{j}(x, D) u, a_{k}(x, D) u\right)\right|$ occurs when $|j-k|$ is large. We give a lemma to handle this case.

LeMma 5.1. If $j, k \geqslant 5$ and $|j-k| \geqslant 4$, then

$$
\left|\left(a_{j}(x, D) u, a_{k}(x, D) v\right)\right| \leqslant C_{t, n}\|a\|^{2} j^{-1-\varepsilon_{t}} k^{-1-\varepsilon_{t}}\|\hat{u}\| L^{2}\left(E_{j}\right)\|\hat{v}\|_{L^{2}\left(E_{k}\right)}
$$

where $u, v \in C_{0}^{\infty}\left(R^{n}\right), \varepsilon_{t}>0$ a constant depending only on $t$ and $C_{t, n}$ is a constant depending only on $n$ and $t$.

Proof. Without loss of generality, we can assume that $j \geqslant k+4, \hat{u} \in C_{0}^{\infty}\left(E_{j}\right)$ and $\hat{v} \in C_{0}^{\infty}\left(E_{k}\right)$. Then

$$
\begin{aligned}
&\left(a_{j}(x, D) u\right.\left., a_{k}(x, D) v\right) \\
&=\left(\frac{1}{2 \pi}\right)^{2 n} \int_{R^{n}} \int_{R^{n}} \int_{R^{n}} e^{i x(\xi-\lambda)} a_{j}(x, \xi) \hat{u}(\xi) \bar{a}_{k}(x, \lambda) \overline{\hat{v}}(\lambda) d \lambda d \xi d x \\
&=\left(\frac{1}{2 \pi}\right)^{2 n}\left(j^{-t} 2^{j}\right)^{n} \int_{R^{n}} \int_{R^{n}} \int_{R^{n}} e^{i x(\xi-\lambda)} a_{j}\left(j^{t} 2^{-j} x, j^{-t} 2^{j} \xi\right) \hat{u}\left(j^{-t} 2^{j} \xi\right) \\
& \times \bar{a}_{k}\left(j^{t} 2^{-j} x, j^{-t} 2^{j} \lambda\right) \overline{\hat{v}}\left(j^{-t} 2^{j} \lambda\right) d \lambda d \xi d x,
\end{aligned}
$$

where the last equality comes from the change of variables $x \rightarrow j^{t} 2^{-j} x, \xi \rightarrow j^{-t} 2^{j} \xi$, $\lambda \rightarrow j^{-t} 2^{j} \lambda$. Integrating with respect to $x$ first and making use of the following identity:

$$
\begin{aligned}
\prod_{s=1}^{n}\left(\frac{1}{1+i\left(\xi_{s}-\lambda_{s}\right) r}\right)\left(\prod_{s=1}^{n}\left(1+(i)^{-r+1} \partial_{x_{s}}^{r}\right)\right)\left(e^{i x(\xi-\lambda)}\right)= & e^{i x(\xi-\lambda)}, \\
& \text { with } r=\left[\frac{(4+t)}{2 t}\right]+1,
\end{aligned}
$$


we have

$$
\begin{aligned}
& \left(a_{j}(x, D) u, a_{k}(x, D) v\right) \\
& \quad=\left(\frac{1}{2 \pi}\right)^{2 n}\left(j^{-t} 2^{j}\right)^{n} \sum_{\alpha \in T}(i)^{(-r+1)|\alpha|}(-1)^{r|\alpha|} \sum_{\beta \leqslant r \alpha} C(r \alpha, \beta) \Gamma_{\alpha, \beta}
\end{aligned}
$$

where

$$
T=\left\{\alpha \in N^{n} \mid \alpha=\left(\alpha_{1}, \ldots, \alpha_{n}\right) \text { and } \alpha_{m}=0 \text { or } 1\right\}
$$

and

$$
\begin{aligned}
I_{\alpha, \beta}=\int_{R^{n}} \int_{R^{n}} \int_{R^{n}} e^{i x(\xi-\lambda)} & \partial_{x}^{r \alpha-\beta}\left(a_{j}\left(j^{t} 2^{-j} x, j^{-t} 2^{j} \xi\right)\right)\left(\prod_{s=1}^{n} \frac{1}{1+i\left(\xi_{s}-\lambda_{s}\right)^{r}}\right) \\
& \times \partial_{x}^{\beta}\left(\bar{a}_{k}\left(j^{t} 2^{-j} x, j^{-t} 2^{j} \lambda\right)\right) \hat{u}\left(j^{-t} 2^{j} \xi\right) \overline{\hat{v}}\left(j^{-t} 2^{j} \lambda\right) d \lambda d \xi d x .
\end{aligned}
$$

Then the lemma follows by an argument similar to the proof of Lemma (4.3) and by the following fact:

If $j \geqslant k+4, r=[(4+t) / 2 t]+1, \varepsilon=r-(4+t) / 2 t$ and $\delta=\left(\delta_{1}, \ldots, \delta_{n}\right) \in N^{n}$ with $\delta_{j}=0$ or 1 , then

$$
\int_{j^{t_{2}-j} E_{j}}\left|\partial_{\xi}^{\delta}\left(\prod_{s=1}^{n} \frac{1}{1+i\left(\xi_{s}-\lambda_{s}\right)^{r}}\right)\right|^{2} d \xi \leqslant C_{n} r^{2 n} j^{-4-2 \varepsilon t} \quad \text { for } \lambda \in j^{t_{2}-j} E_{k},
$$

where $C_{n}$ is a constant depending only on $n$.

REMARK. Let $\Phi, \varphi$ be two positive functions defined on $R^{n} \times R^{n}$. We say $a$ is in the Beals-Fefferman class $S_{\Phi, \varphi}^{0,0}$, if $a \in C^{\infty}\left(R^{n} \times R^{n}\right)$ and there exists a constant $C_{\alpha, \beta}>0$ such that

$$
\begin{aligned}
& \left|\partial_{x}^{\alpha} \partial_{\xi}^{\beta} a(x, \xi)\right| \leqslant C_{\alpha, \beta}(\Phi(x, \xi))^{-|\beta|}(\varphi(x, \xi))^{-|\alpha|}, \\
& \qquad \text { for }(x, \xi) \in R^{n} \times R^{n}, \alpha, \beta \in N^{n} .
\end{aligned}
$$

In Hörmander [8], it is proved that $a(x, D)$ is continuous from $L^{2}\left(R^{n}\right)$ to $L^{2}\left(R^{n}\right)$ if $a \in S_{\Phi, \varphi}^{0,0}$ and $\Phi, \varphi$ satisfy the following conditions:

(1) There exists $\varepsilon_{0}>0, C_{0}>0$ such that if $(x, \xi),(y, n) \in R^{n} \times R^{n}$ and

$$
\frac{|x-y|}{\varphi(x, \xi)}+\frac{|\xi-n|}{\Phi(x, \xi)}<\varepsilon_{0},
$$

then

$$
C_{0}^{-1}<\frac{\varphi(y, n)}{\varphi(x, \xi)}<C_{0} \quad \text { and } \quad C_{0}^{-1}<\frac{\Phi(y, n)}{\Phi(x, \xi)}<C_{0} .
$$

(2) $\Phi(x, \xi) \cdot \varphi(x, \xi) \geqslant 1$, for $(x, \xi) \in R^{n} \times R^{n}$.

(3) There exist $C>0, t>0$ such that

$$
\frac{\varphi(y, n)}{\varphi(x, \xi)}+\frac{\Phi(y, n)}{\Phi(x, \xi)} \leqslant C(1+\Phi(x, \xi)|x-y|+\varphi(x, \xi)|\xi-n|)^{t},
$$

$$
\text { for }(x, \xi),(y, n) \in R^{n} \times R^{n} \text {. }
$$


We now construct a symbol $a_{0}$ which is in $C^{\infty}(R \times R)$ and satisfies the following condition:

For any $\alpha, \beta \in N$, there exists a constant $C_{\alpha, \beta}>0$ such that

$$
\left|\partial_{x}^{\alpha} \partial_{\xi}^{\beta} a(x, \xi)\right| \leqslant C_{\alpha, \beta}\left((1+|\xi|)(\log (1+|\xi|))^{-1}\right)^{|\alpha|-|\beta|},
$$

$(x, \xi) \in R \times R$. Suppose such an $a_{0}$ exists. Then by Theorem $4, a_{0}(x, D)$ is continuous from $L^{2}(R)$ to $L^{2}(R)$. Also, we can see that $a_{0} \in S_{\Phi_{0}, \varphi_{0}}^{0,0}$ with $\Phi_{0}(x, \xi)$ $=(1+|\xi|)(\log (1+|\xi|))^{-1}$ and $\varphi_{0}(x, \xi)=(1+|\xi|)^{-1} \log (1+|\xi|)$, which can not satisfy (3). Therefore (1), (2) and (3) are not the necessary conditions for the $L^{2}$ continuity.

Construction of $a_{0}$. Let $\varphi \in C_{0}^{\infty}(R)$ such that $\varphi \geqslant 0, \varphi(0)=1$ and $\operatorname{supp} \Phi \subseteq\{|\xi|$ $\leqslant 1\}$. We define

$$
a_{0}(x, \xi)=\sum_{j=4}^{\infty} e^{i j^{-1} 2^{j} x} \varphi\left(j 2^{-j}\left(\xi-2^{j}\right)\right), \quad(x, \xi) \in R \times R .
$$

Then $a_{0}$ satisfy the condition that we want.

6. Proof of Theorem 5. We denote a constant depending only on $\rho$ by $C_{\rho}$, which may vary from time to time. At first, we prove the following lemma.

LEMMA 6.1. If $0<\rho<1$, then

$$
\left|\left(a_{j}(x, D) u, a_{k}(x, D) v\right)\right| \leqslant C_{\rho} 2^{-j \varepsilon_{\rho}} 2^{-k \varepsilon_{\rho}}\|\hat{u}\|_{L^{2}\left(E_{j}\right)}\|\hat{v}\|_{L^{2}\left(E_{k}\right)},
$$

where $u, v \in C_{0}^{\infty}(R), j, k \geqslant 1,|j-k| \geqslant 4$ and $\varepsilon_{\rho}=(1-\rho) / 4$.

Proof. By following the proof of Lemma 4.3, we have

$$
\left(a_{j}(x, D) u, a_{k}(x, D) v\right)=\left(\frac{1}{2 \pi}\right)^{2} 2^{j \rho} \sum_{\alpha \in T} \sum_{\beta \leqslant \alpha}(-1)^{\alpha} I_{\alpha, \beta},
$$

where $T=\{0,1\}$ and

$$
\begin{aligned}
I_{\alpha, \beta}=\int_{R} \int_{R} \int_{R} e^{i x(\xi-\lambda)} \partial_{x}^{\alpha-\beta}\left(a_{j}\left(2^{-j \rho} x, 2^{j \rho} \xi\right)\right) \\
\quad \times \partial_{x}^{\beta}\left(\bar{a}_{k}\left(2^{-j \rho} x, 2^{j \rho} \lambda\right)\right)\left(\frac{1}{1+i(\xi-\lambda)}\right) u\left(2^{j \rho} \xi\right) \overline{\hat{v}}\left(2^{j \rho} \lambda\right) d \lambda d \xi d x .
\end{aligned}
$$

By the proof of Lemma 4.3, we have

$$
\left|I_{\alpha, 0}\right| \leqslant C_{\rho} 2^{-j \varepsilon_{\rho}} 2^{-k \varepsilon_{\rho}}\|\hat{u}\|_{L^{2}\left(E_{j}\right)}\|\hat{v}\|_{L^{2}\left(E_{k}\right)} \quad \text { with } \alpha=0 \text { or } 1 .
$$

To estimate $I_{0,1}$, we use a technique similar to the proof of Lemma 4.3, but we integrate with respect to $\lambda$ instead of $\xi$.

We state the following results and omit their proofs.

Lemma 6.2. If $-\infty<\rho<0$, then

$$
\left|\left(a_{j}(x, D) u, a_{k}(x, D) v\right)\right| \leqslant C_{\rho} 2^{-j / 4} 2^{-k / 4}\|\hat{u}\|_{L^{2}\left(E_{j}\right)}\|\hat{v}\|_{L^{2}\left(E_{k}\right)},
$$

where $u, v \in C_{0}^{\infty}(R), j, k \geqslant 1$ and $|j-k| \geqslant 4$. 
Theorem 5 follows from Lemmas 4.1, 4.2, 6.1, and 6.2.

By using arguments similar to the proofs of Theorem 4 and Theorem 5, we obtain Corollary 5.1.

\section{REFERENCES}

1. A. Calderon and R. Vaillancourt, On the boundedness of pseudodifferential operators, Proc. Nat. Acad. Sci. U.S.A. 69 (1972), 1185-1187.

2. R. R. Coifman and Y. Meyer, Au delá des opérators pseudo-differentials, Asterisque 57 (1978).

3. T. Kato, Boundedness of some pseudodifferential operators, Osaka J. Math. 13 (1976), 1-9.

4. H. Kumano-go, Pseudodifferential operators, MIT Press, Cambridge, Mass., 1981.

5. R. Beals, On the boundedness of pseudo-differential operators, Comm. Partial Differential Equations 2 (1977), 1063-1070.

6. H. O. Cordes, On compactness of commutators of multiplications and convolutions, and boundedness of pseudodifferential operators, J. Funct. Anal. 18 (1975), 115-131.

7. T. Muramato and M. Nagase, $L^{2}$-boundedness of pseudodifferential operators with non-regular symbols, Canad. Math. Soc. Conf. Proc., Vol. 1, Amer. Math. Soc., Providence, R.I., 1981, p. 138.

8. L. Hörmander, The analysis of linear partial differential operators. III, Springer-Verlag , 1985, p. 75 and Chapter 18.

Department of Mathematics, Purdue University, West Lafayette, Indiana 47907 